\title{
Complete Atrioventricular Block Complicating Hypothyrodism
}

\author{
Ratchaneewan Kwancharoen, Swangjit Suraamornkul, Petch Rawdaree
}

Endocrinology and Metabolism Unit, Bangkok Metropolitan Medical College and Vajira Hospital, Bangkok, Thailand

\begin{abstract}
An 85-year old Thai woman was admitted to the hospital for dyspnea. Her ECG showed complete atrioventricular (AV) block. Her laboratory data upon admission revealed low serum thyroxine level and elevated thyrotropin level. Levothyroxine was started, and the AV conduction block was supported by a temporary pacemaker. At the end of the second week, her free T4 fell within normal range. The temporary pacemaker was subsequently removed. She was discharged without permanent pacing. This case demonstrated that hypothyroidism can cause complete atrioventricular block. Levothyroxine replacement can restore AV conduction in the hypothyroid patient.
\end{abstract}

Key words: hypothyroidism, atrioventricular block

Hypothyroidism can cause a variety of manifestations, including cardiovascular dysfunction. The more common presenting signs are sinus bradycardia and pericardial effusion. ${ }^{1}$ The affected patient usually has severe symptoms. Hypothyroidism rarely causes complete atrioventricular (AV) block. ${ }^{2}$ In this paper, we report a case presenting with mild symptoms of hypothyroidism but with the uncommon manifestation of complete AV block. The heart block was subsequently treated with thyroid hormone replacement.

\section{Case Report}

A previously well 85-year-old Thai woman consulted for a two-month history of dyspnea. The dyspnea was constant and not apparently aggravated by exertion. She also noted associated leg edema, constipation, cold intolerance and dry skin. She did not experience orthopnea, paroxysmal nocturnal dyspnea, chest pain, syncope, urinary symptoms or unusual weight loss. She had hypertension and dyslipidemia for approximately 2 years, but had no maintenance medication. She did not have any history of thyroid problems; heart disease; and irradiation to, or surgery to the neck area.

On physical examination, she was conscious and cooperative but appeared tired. Her vital signs were as follows: temperature $36.7^{\circ} \mathrm{C}$, blood pressure $120 / 90$, pulse rate 40 beats/minute, and respiratory rate 18 breaths/minute. She had coarse hair, facial puffiness and thinning of the lateral third of the eyebrows. She had no macroglossia. The right lobe of the thyroid gland was not palpable, while the left lobe was firm and mildly enlarged. No superficial lymphadenopathy was detected. Careful examination of the cardiovascular system revealed bradycardia; normal S1 and S2; and no cardiac murmur. Examination of the extremities showed dry, cracked skin

ISSN 0857-1074

Copyright $(2011$ by the JAFES

Received January 24, 2011. Accepted March 16, 2011. with no apparent yellowing of the palms. She had full and slow pulses, and grade 1 bipedal pitting edema. Neuromuscular examination revealed slow relaxation of reflexes.

Laboratory results were as follows: fasting plasma glucose $88 \mathrm{mg} / \mathrm{dL}$, blood urea nitrogen $17 \mathrm{mg} / \mathrm{dL}$, and serum creatinine $1.4 \mathrm{mg} / \mathrm{dL}$. Her lipid profile revealed a total cholesterol level of $180 \mathrm{mg} / \mathrm{dL}$, low-density lipoprotein cholesterol (LDL-C) of $114 \mathrm{mg} / \mathrm{dL}$, high-density lipoprotein (HDL-C) of $31 \mathrm{mg} / \mathrm{dL}$ and triglyceride level of $154 \mathrm{mg} / \mathrm{dL}$. Troponin-T level was within normal limits. Her thyroid function tests revealed elevated thyroid stimulating hormone $(\mathrm{TSH})$ (> $75 \mathrm{mIU} / \mathrm{L}$, normal value 0.4 to $4.0 \mathrm{mIU} / \mathrm{L})$, low free $\mathrm{T} 3(1.0 \mathrm{pg} / \mathrm{mL}$, normal value 2.0 to $4.0 \mathrm{pg} / \mathrm{mL})$, and low free $\mathrm{T} 4(<0.30 \mathrm{ng} / \mathrm{dL}$, normal value 1.0 to $2.0 \mathrm{ng} / \mathrm{dL}$ ). Antithyroglobulin antibody (anti-TG Ab) was > 3,000 IU (normal value 0 to $40 \mathrm{IU}$ ), and antithyroperoxidase antibody (anti-TPO Ab) was > $100 \mathrm{IU}$ (normal value 0 to $35 \mathrm{IU}$ ).

Her chest $x$-ray demonstrated normal lung parenchyma and thoracic cage, and mild cardiomegaly. A 12-lead electrocardiogram (ECG) showed complete heart block (Figure 1).

She underwent temporary pacemaker insertion with an output of $40 \mathrm{~mA}$ and a set rate 60 beats/minute. The ECG done after the procedure is shown in Figure 2.

On admission, the patient was started on levothyroxine at a dose of $25 \mathrm{mcg} /$ day. This dose was adjusted by increments of $25 \mathrm{mcg}$ every three days until a dose of 100 $\mathrm{mcg} /$ day was reached by the tenth hospital day. Her heart rate increased, and some electrical activity was found to be conducted through the atrioventricular node as shown in Figure 3.

Corresponding author: Ratchaneewan Kwancharoen, $M D$

Endocrinology and Metabolism unit, Bangkok Metropolitant

Medical Collage and Vajira Hospital,Bangkok, Thailand.

E-mail: ratch.kwn@gmail.com 


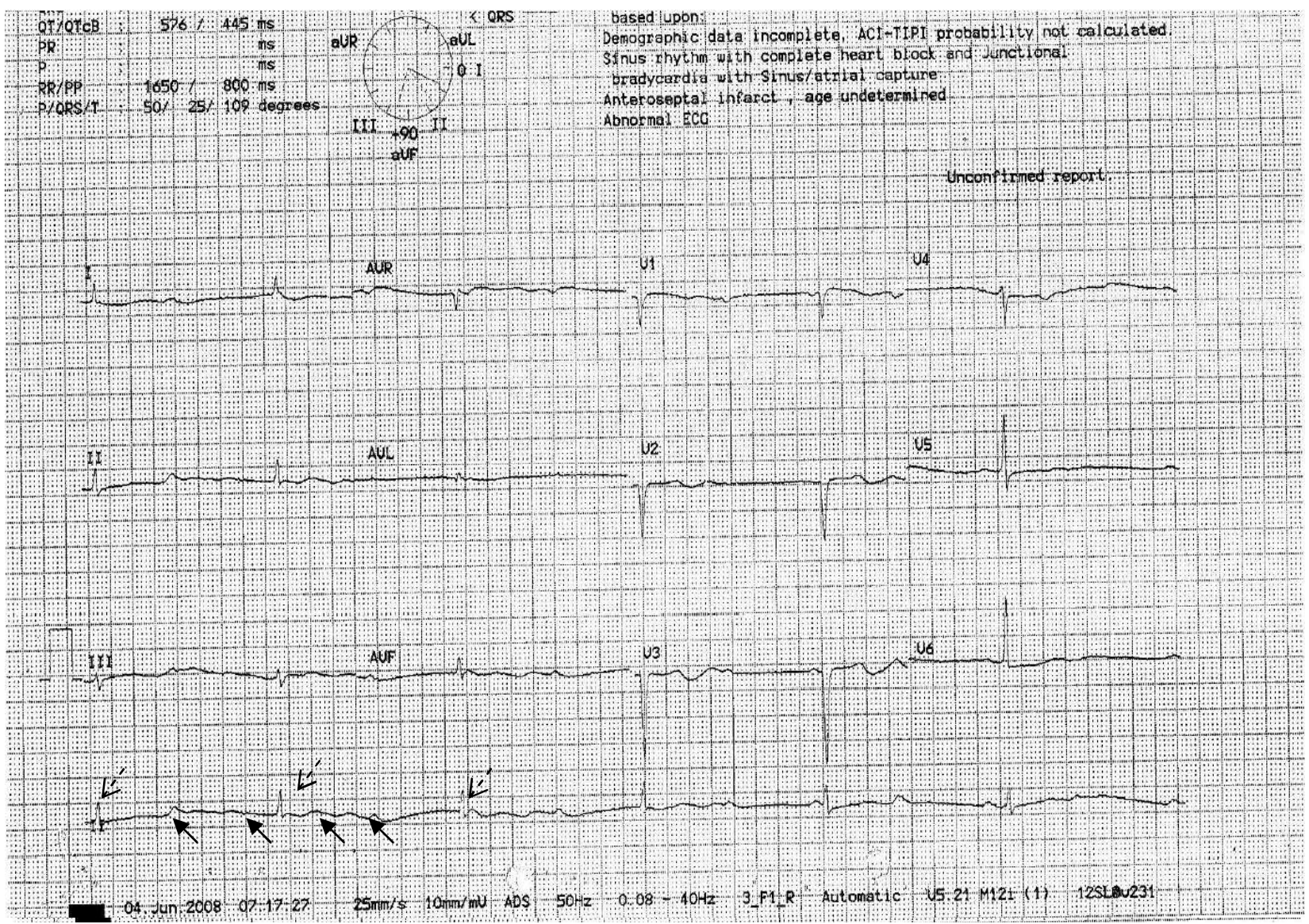

Figure 1. Complete heart block pattern on 12-lead ECG showing unconducted $P$ waves (bold arrows) and idioventricular rhythm (dashed arrows).

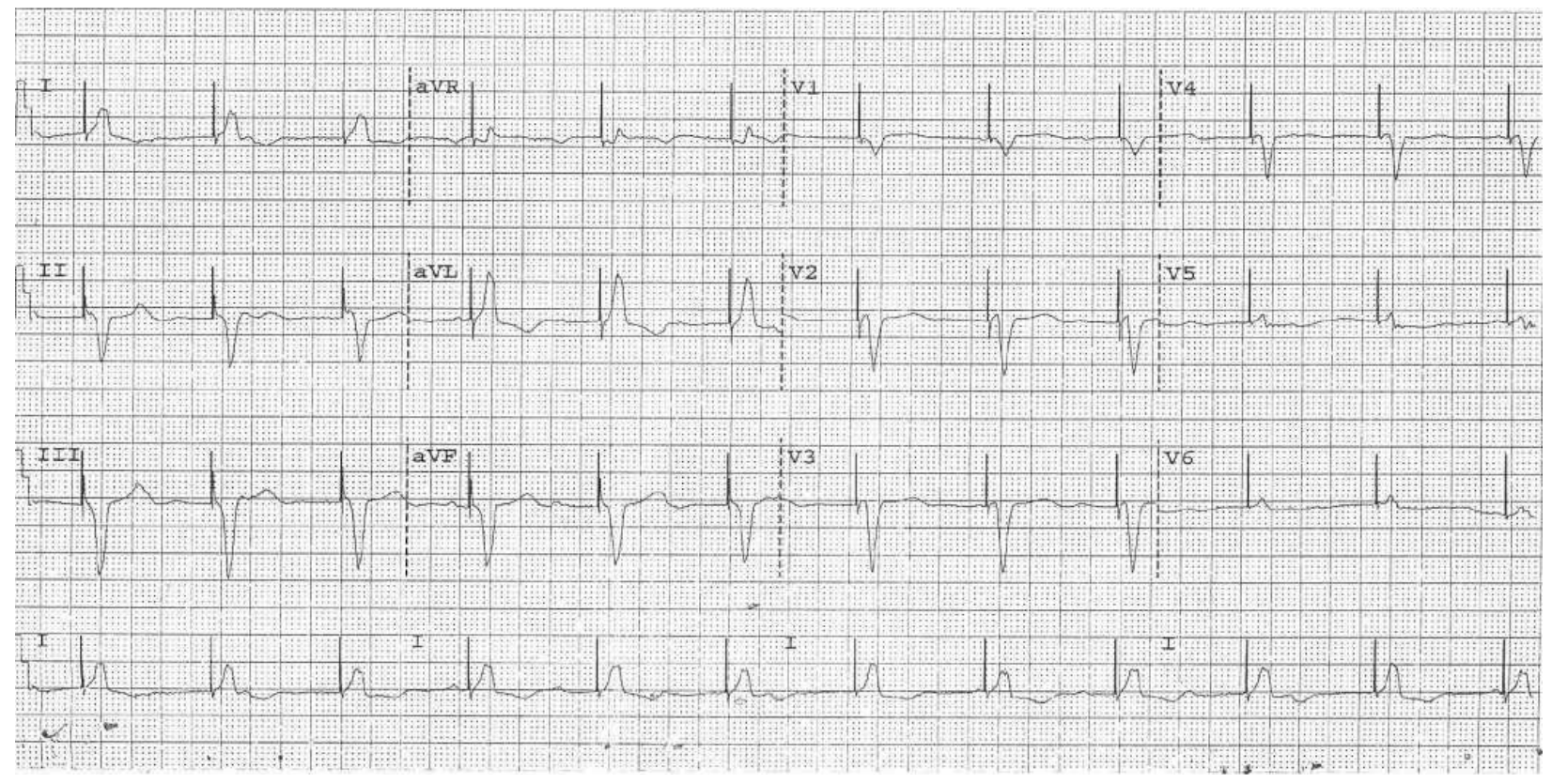

Figure 2. Twelve-lead ECG after temporary pacemaker insertion.

On the second week of levothyroxine replacement, her free T4 level became normal $(1.04 \mathrm{pg} / \mathrm{mL})$. Her ECG was compatible with a Mobitz type I atrioventricular block (Figure 4). The temporary pacemaker was subsequently removed. No new conduction blocks were observed on close monitoring during the following week. She was discharged on the third week of her hospital stay.

The patient returned for follow-up 2 weeks after discharge. She did not experience dizziness, dyspnea or syncope. Subsequent ECGs done (Figures 4 and 5) shows 
persistence of the same Mobitz type I AV block pattern. Free T4 level was within normal $(1.54 \mathrm{pg} / \mathrm{mL})$.

\section{Discussion}

Thyroid hormones affect various organs throughout the body, particularly the cardiovascular and autonomic systems. There are three main physiologic mechanisms by which thyroid hormones affect the heart. First, triiodothyronine (T3) binds to the $\mathrm{T} 3$ receptor in the nucleus of the cardiac myocyte and causes specific gene expression. The second mechanism is via the effect of T3 on the sensitivity of the sympathetic nervous system. Third, T3 causes changes in the peripheral circulatory system that results in an increase in cardiac filling and changes in cardiac contractility.

Thyroid hormones also play an important role in electrical current generation and conduction in the myocardium. Triiodothyronine increases systolic depolarization and decreases diastolic repolarization. It shortens the duration of the action potential, the refractory period of the atrial myocardium and the atrioventricular nodal period. These changes are caused by increase in sodium pump density and sodium and potassium permeability. For the electrical current generation, T3 causes an increase in the L-type calcium channel at the sinu-atrial node. ${ }^{2}$

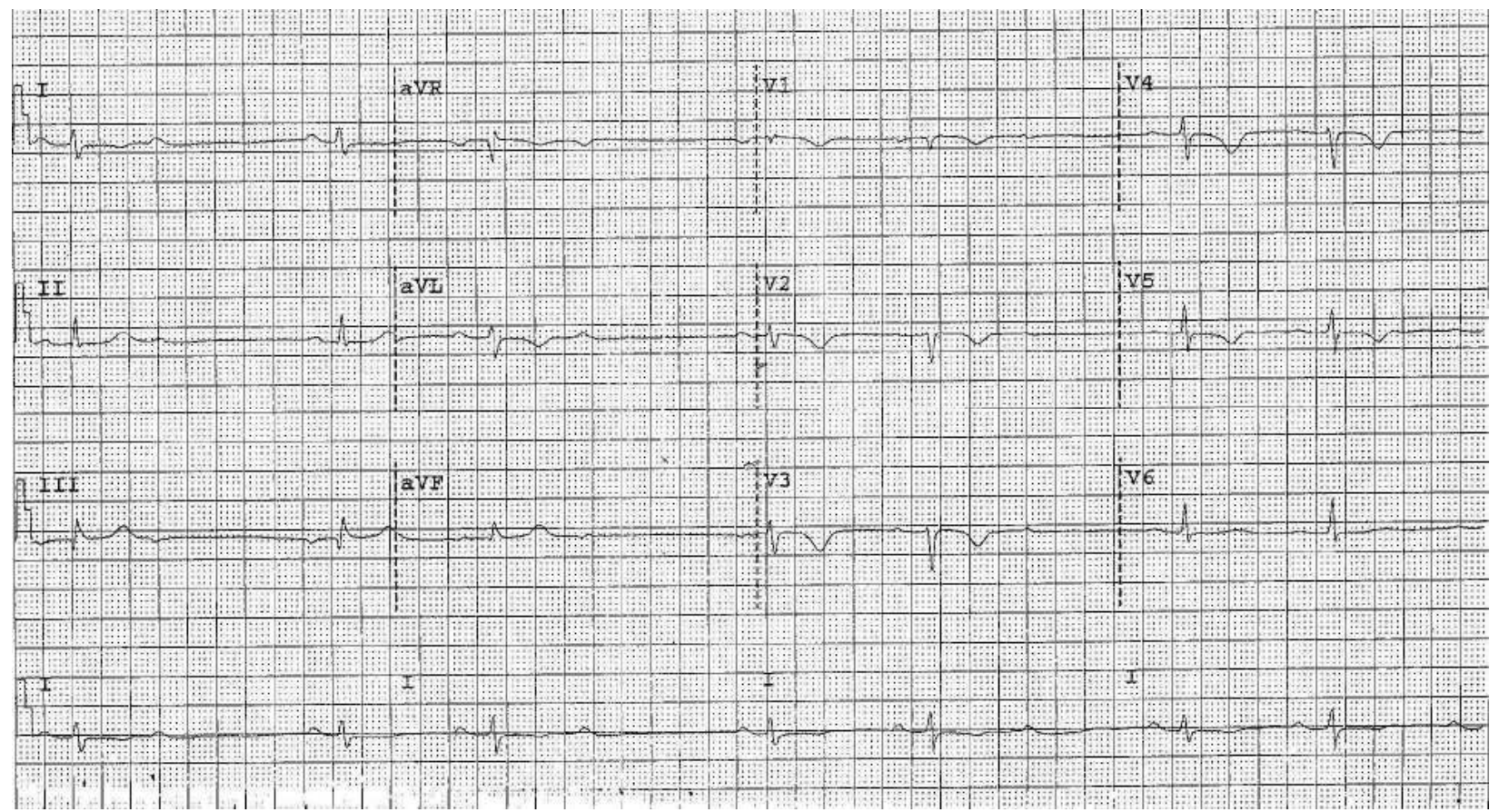

Figure 3. Twelve-lead ECG after 10 days of levothyroxine treatment.

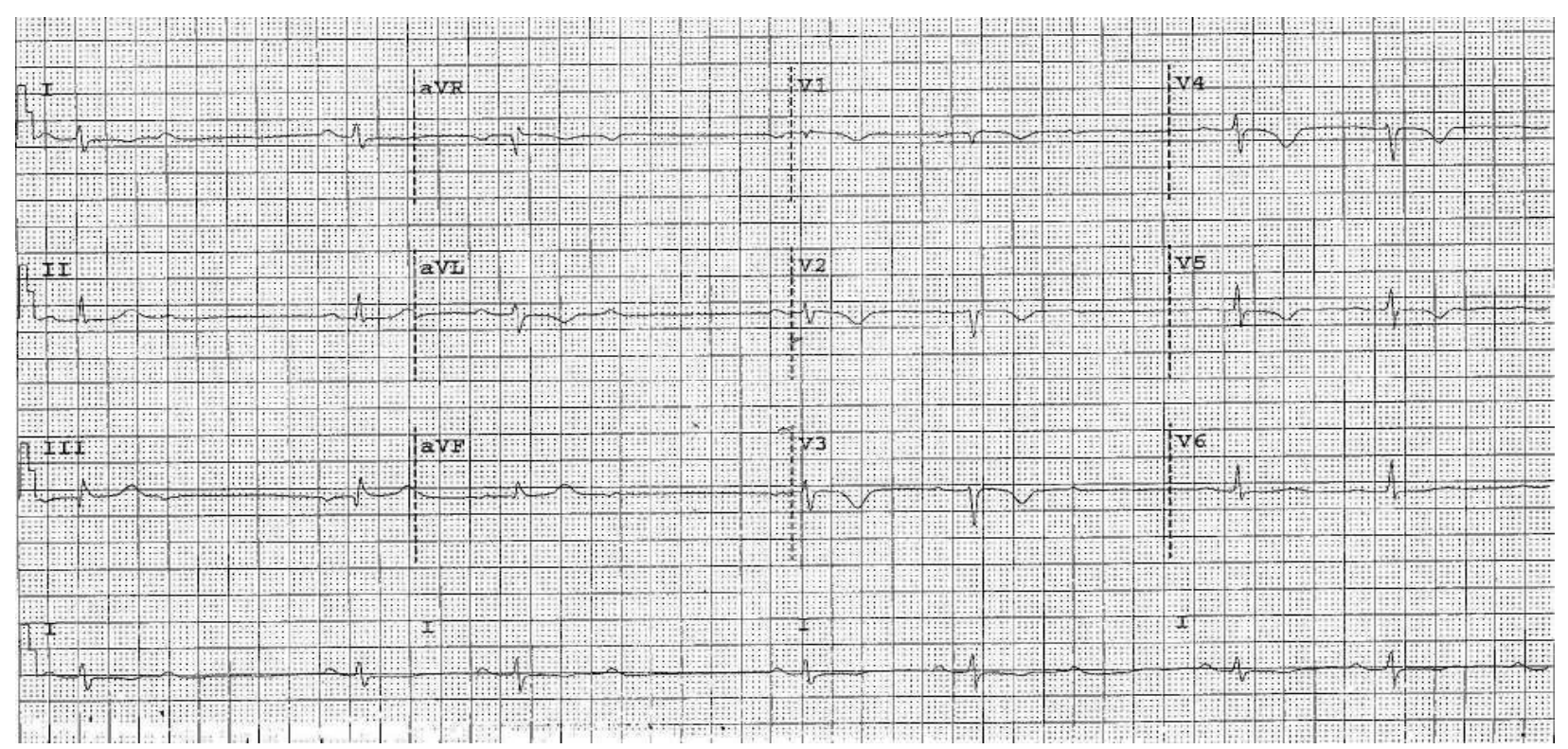

Figure 4. Twelve-lead ECG after 21 days of levothyroxine treatment. 


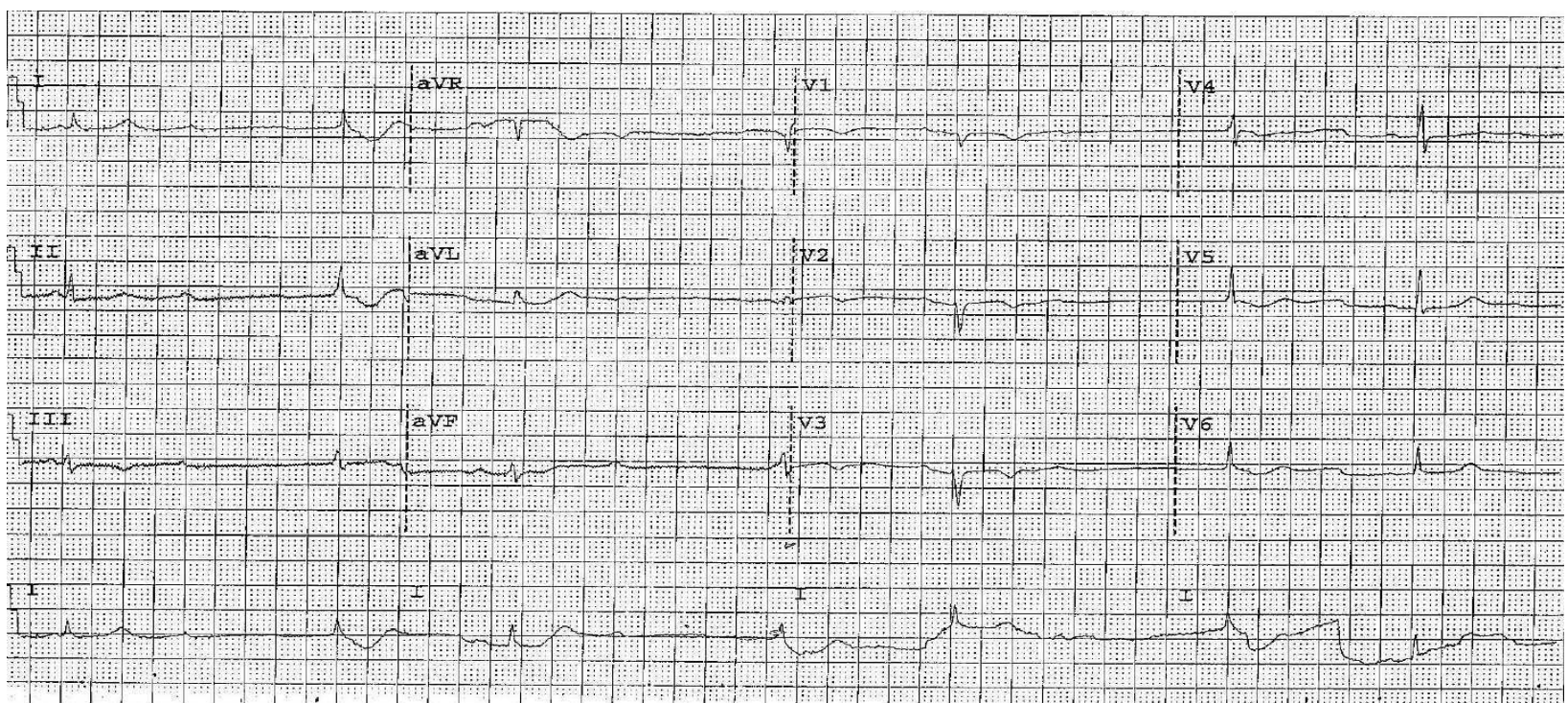

Figure 5. Twelve-lead ECG on the fifth week of levothyroxine treatment.

The cardiac electrical activity changes that can be observed in hypothyroid patients are sinus bradycardia, QT interval prolongation, $\mathrm{T}$ wave inversion and $\mathrm{AV}$ block. Other cardiovascular effects include increased peripheral vasoconstriction; decreased cardiac contractility; and prolonged diastolic relaxation. Subsequently, cardiac output decreases due to a reduction in stroke volume and heart rate. Changes ensue as a result of the decrease in inotropy and chronotropy, such as narrowing of pulse pressure, prolongation of circulatory time and decrease in peripheral blood flow. ${ }^{3-5}$

The estimated prevalence of hypothyroidism varies, depending on the population studied. In the United States, hypothyroidism affects approximately $0.3 \%$ of the general population. ${ }^{1}$ Among healthy elderly individuals, prevalence rates range from 0.55 to $1.5 \% .^{6-7}$ Moreover, asymptomatic hypothyroidism rarely causes cardiovascular morbidity and mortality. Although the AV node is the first part of the AV conduction system to be affected by aging, the prevalence of second and third degree blocks in the general population is only approximately $1 \% .^{8-9}$

In this case, the patient had been well until a few weeks before admission, and had never had any prior arrhythmic event. Most case reports of hypothyroid patients with conduction abnormalities describe severe accompanying symptoms, such as myxedema coma. In the absence of these symptoms, conduction abnormality is usually accompanied by other cardiac conditions, such as cardiomegaly or pericardial effusion. ${ }^{10-12}$ In contrast, the patient had mild to moderate symptoms of hypothyroidism, but had a severe cardiovascular manifestation of complete AV block.

In previous case reports, conduction blocks were reversed by thyroxine replacement after a few days to a few weeks. Although the patient received an adequate dose of thyroid hormone and became subsequently euthyroid, she did not revert to sinus rhythm. Her ECG tracing revealed a Mobitz type I pattern which was not clinically significant and did not require any treatment. The patient may have had an underlying Mobitz type I abnormality. Finally, she was discharged without the need for a pacemaker.

In conclusion, this case demonstrates an uncommon presentation of hypothyroidism that can cause complete atrioventricular block. The AV block was treated with appropriate diagnosis and management. With prompt recognition and treatment, we were able to avoid invasive procedures and minimize morbidity.

\section{References}

1. Brent G, Davies TF, Larsen PR. Hypothyroidism and thyroiditis. In: Kronenberg HM, Melmed S, Polonsky KS, Larsen PR, eds. Williams Textbook of Endocrinology. $11^{\text {th }}$ ed. Philadephia: Saunders Elsevier; 2008:377-409.

2. Freedberg AS, Papp JG, Williams EMV. The effect of altered thyroid state on atrial intracellular potentials. J Physiol. 1970 Apr;207(2):35769.

3. Kahaly GJ, Dillmann WH. Thyroid hormone action in the heart. Endocr Rev. 2005 Aug;26(5):704-28.

4. Klein I, Ojamaa K. Thyroid hormone and the cardiovascular system. N Engl J Med. 2001 Feb 15;344(7):501-9.

5. Kahaly GJ. Cardiovascular and athrogenic aspects of subclinical hypothyroidism. Thyroid. 2000 Aug;10(8):665-79.

6. Graziano Cet al., Thyroid function abnormalities and cognitive impairment in the elderly: Results of the Invecchiare in Chianti study. J Am Geriatr Soc. 2009 Jan;57(1):89-93.

7. Cappola AR, et al., Thyroid status, cardiovascular risk, and mortality in older adults. JAMA. 2006 Mar 1;295(9):1033-41.

8. Schoenmakers N, de Graaff WE, Peters RH. Hypothyroidism as the cause of atrioventricular block in an elderly patient. Neth Heart J. 2008 Feb;16(2):57-9.

9. Fragakis N, Katsaris G. Arrhythmias in the elderly: Modern management. Hellenic J Cardiol. 2006 Mar-Apr;47(2):84-92.

10. Singh JB, et al., Reversible atrioventricular block in myxedema. Chest. 1973 Apr;63(4):582-5.

11. Schantz ET, Dubbs AW. Complete auriculoventricular block in myxedema with reversion to normal sinus rhythm on thyroid therapy. Am Heart J. 1951 Apr;41(4):613-9.

12. Nakayama $\mathrm{Y}$, et al., A case of transient 2:1 atrioventricular block, resolved by thyroxine supplementation for subclinical hypothyroidism. Pacing Clin Electrophysiol. 2006 Jan;29(1):106-8. 